\title{
Quantifying myocardial inflammation using F18-fluorodeoxyglucose positron emission tomography in cardiac sarcoidosis
}

\author{
Alfonso $\mathrm{H}$. Waller, $M D^{a},{ }^{a}$ and Ron Blankstein, $M^{a}$ \\ ${ }^{a}$ Non-Invasive Cardiovascular Imaging Program, Departments of Medicine (Cardiovascular \\ Division) and Radiology, Brigham and Women's Hospital, Boston, MA
}

Received May 20, 2014; accepted May 20, 2014

doi: $10.1007 / \mathrm{s} 12350-014-9921-5$

\section{See related article, pp. 925-939}

Sarcoidosis is a disease of unknown etiology which is characterized by the formation of non-caseating granulomas in multiple organs. ${ }^{1}$ Cardiac involvement in sarcoidosis, which occurs in at least one in four patients, ${ }^{2}$ is associated with a worse prognosis and significant morbidity from conduction abnormalities, arrhythmias, and congestive heart failure. Nevertheless, the diagnosis of cardiac sarcoidosis (CS) is often challenging, and relies on integrating both clinical and imaging findings. In addition to diagnosis, advanced imaging techniques such as cardiac magnetic resonance imaging (CMR) and cardiac positron emission tomography (PET) are now also being used to determine the risk of future adverse events, ${ }^{3}$ to identify which patients are most likely to benefit from immunosuppressive therapy, and to monitor response to therapy. ${ }^{4}$

Cardiac PET imaging for CS includes a rest myocardial perfusion imaging scan (using either ${ }^{13} \mathrm{~N}$ Ammonia or ${ }^{82}$ Rubidium) and an ${ }^{18}$ F-fluorodeoxyglucose (FDG) scan to identify areas of myocardial inflammation. ${ }^{5}$ Interpretation of the cardiac PET study is performed using a qualitative assessment with the perfusion and FDG images being simultaneously displayed (see Figure 1 for example). Rest myocardial perfusion defects may represent areas of scar related to fibrosis or

Reprint requests: Ron Blankstein, MD, Non-Invasive Cardiovascular Imaging Program, Departments of Medicine (Cardiovascular Division) and Radiology, Brigham and Women's Hospital, 75 Francis Street Room Shapiro 5096, Boston, MA 02115; rblankstein@mgh. harvard.edu; rblankstein@partners.org

J Nucl Cardiol 2014;21:940-3.

$1071-3581 / \$ 34.00$

Copyright (C) 2014 American Society of Nuclear Cardiology. areas of decreased perfusion from inflammation causing compression of the microvasculature. ${ }^{3}$ Following suppression of glucose uptake from the normal myocardium, and in the absence of coronary heart disease, which can be associated with hibernating myocardium, focal areas of FDG uptake represent active myocardial inflammation. Importantly, such abnormalities are not only useful for establishing the diagnosis of CS, but provide important prognostic information regarding a patient's future risk of death or ventricular tachycardia. ${ }^{3}$ However, a limitation of FDG PET scans is that diffuse uptake of FDG may be visualized which is non-specific and due to either incomplete suppression of FDG from normal areas of myocardium vs diffuse inflammation. Such results-which despite ideal patient preparation occur $\sim 10 \%-15 \%$ of the time-may lead to inconclusive study results. However, there are no established quantitative methods that can reliably distinguish patients with diffuse FDG from inflammation related to CS from those who have benign uptake. Tahara et $\mathrm{al}^{6}$ have shown that heterogeneous myocardial FDG uptake is more common in CS patients and further data are needed regarding the discriminatory performance of this parameter among patients who have inconclusive FDG scans.

To date, there is relatively little data on quantitative assessment of CS by PET imaging-with some studies evaluating the intensity of FDG uptake and only one prior study evaluating the extent of myocardial inflammation. ${ }^{4}$ The intensity of FDG uptake on PET can be measured by standardized uptake value (SUV), where a reader identifies voxels with FDG uptake, and the voxel with the highest intensity of FDG is used to define the $\mathrm{SUV}_{\text {max }}$. The extent of FDG (e.g., SUV volume or volume of inflammation) can be measured by identifying voxels with an SUV intensity above a pre-defined threshold value. ${ }^{4,7}$

In this issue of the Journal of Nuclear Cardiology, Ahmadian et $\mathrm{al}^{8}$ propose a new method to quantify the 


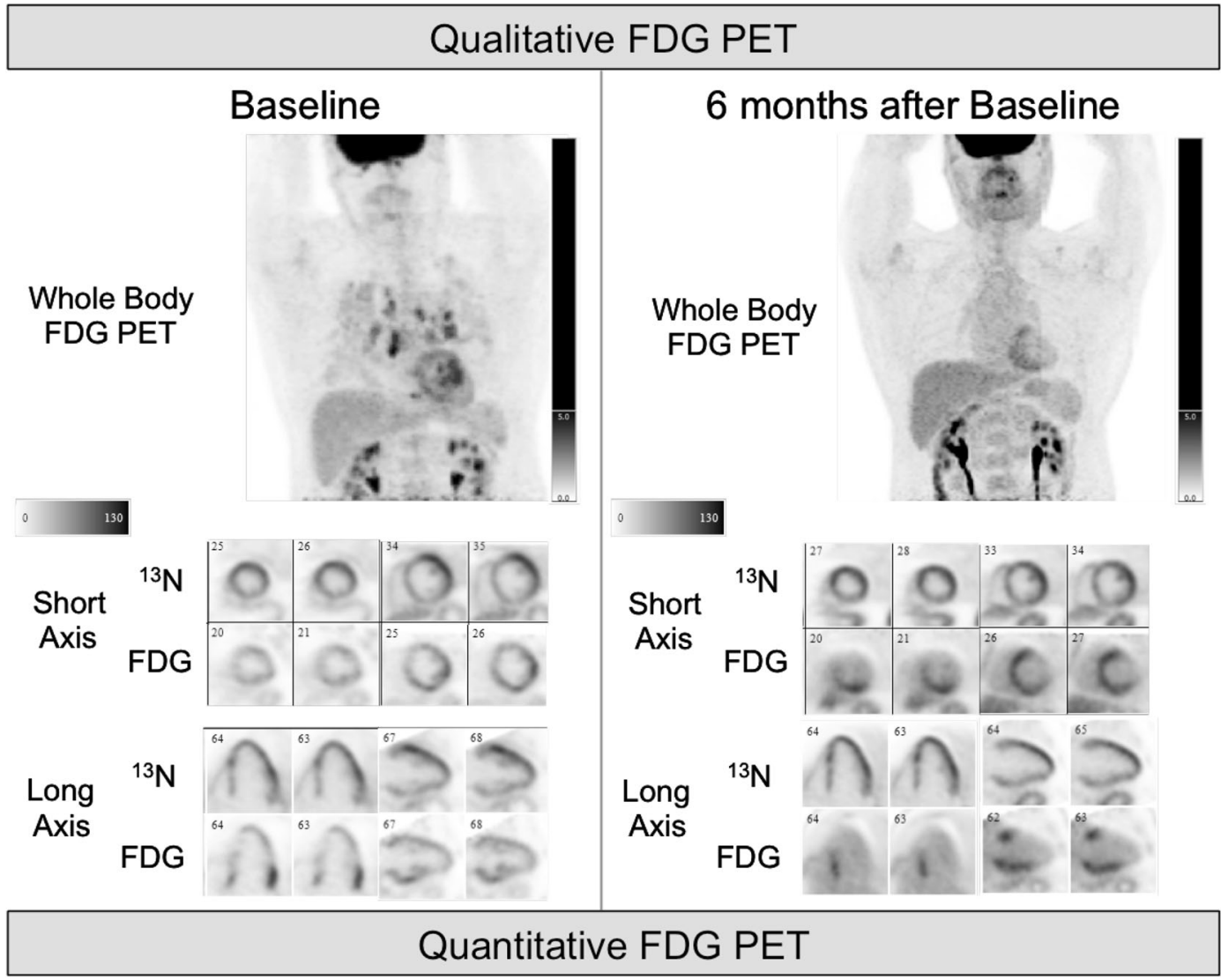

\begin{tabular}{|c|c|c|c|c|c|c|}
\hline & $\begin{array}{c}\text { SUV } \\
\text { Max }\end{array}$ & $\begin{array}{c}\text { SUV } \\
\text { LVBP }\end{array}$ & $\begin{array}{c}\text { SUV volume w/ } \\
1.5 \times \text { SUV } \\
\text { SUV threshold } \\
\text { (Ahmadian A et al) }\end{array}$ & $\begin{array}{c}\text { SUV volume w/ } \\
2.7 \text { SUV } \\
\text { threshold } \\
\text { (Osborne MT et al) }\end{array}$ & $\begin{array}{c}\text { SUV volume w/ } \\
4.1 \text { SUV } \\
\text { threshold } \\
\text { (Osborne MT et al) }\end{array}$ & $\begin{array}{c}\text { Qualitative } \\
\text { Assessment }\end{array}$ \\
\hline Baseline & 6.74 & 1.76 & $90.97 \mathrm{~cm}^{3}$ & $88.24 \mathrm{~cm}^{3}$ & $9.84 \mathrm{~cm}^{3}$ & ++ \\
\hline $\begin{array}{c}6 \text { months } \\
\text { after } \\
\text { Baseline }\end{array}$ & 4.37 & 1.70 & $29.68 \mathrm{~cm}^{3}$ & $20.19 \mathrm{~cm}^{3}$ & $0.22 \mathrm{~cm}^{3}$ & +++ \\
\hline$\%$ Change & $\downarrow 35 \%$ & - & $\Downarrow 67 \%$ & $\Downarrow 77 \%$ & $\Downarrow 98 \%$ & $\uparrow$ \\
\hline
\end{tabular}

Figure 1. Serial FDG PET exams showing changes in inflammation. A 62-year-old man with cardiac and extra-cardiac sarcoidosis was imaged at baseline and after treatment with corticosteroids. The whole body FDG images demonstrate a decrease in extra-cardiac activity. However on the dedicated myocardial rest perfusion with ${ }^{13} \mathrm{~N}$-Ammonia $\left({ }^{13} \mathrm{~N}\right)$ and dedicated cardiac FDG imaging, visually there appears to be new areas of FDG activity and progression of disease. While quantitative assessment of FDG measurements by SUVmax $(\mathrm{g} / \mathrm{mL})$ and SUV volume $\left(\mathrm{cm}^{3}\right)$ was obtained using the Ahmadian et $\mathrm{al}^{8}$ and the Osborne et $\mathrm{al}^{4}$ methods, demonstrate a reduction in FDG.

amount of FDG uptake in the myocardium among patients undergoing cardiac PET imaging for suspected or known CS. When would such quantification techniques be most clinically useful?

The assessment of FDG uptake represents a "hot spot" imaging technique whereby there is increased tracer uptake in abnormal areas. This method relies on normalizing tracer uptake to pixels with the highest intensity and creates images that display relative differences in tracer distribution. When there is focal FDG uptake, the maximal intensity pixel in the heart corresponds to the most abnormal activity. Therefore, when only mildly abnormal areas are present, normalization could amplify the severity of disease, a problem which is 
particularly important when comparing serial scans to monitor the response to immunosuppressive therapy. Consider the example of the patient shown in Figure 1 who was treated with corticosteroids and was referred for a cardiac PET to assess the response to therapy. A qualitative visual read of the cardiac images comparing the baseline study with the 6 month follow-up scan (see FDG short axis and long axis images) may be interpreted as showing increased FDG uptake following therapy. However, when four different quantitative techniques are used in this case, there is a reduction in the intensity (i.e., SUV max) and extent (i.e., volume) of inflammation indicating that this patient has a favorable, albeit incomplete, response to therapy.

Quantitative assessment in imaging for CS may eliminate normalization errors, reduce interobserver bias from semi-quantitative or qualitative interpretations, and provide a means to better quantify disease activity. Such a quantitative assessment is most important when monitoring changes in FDG uptake on serial studies performed to monitor the response to therapy. However, even when quantified, the change in FDG uptake should be interpreted carefully. While a significant decrease in inflammation often represents favorable response to therapy (particularly if also accompanied by an improvement in ejection fraction or a decrease in the size and/or intensity of resting perfusion defects), in some patients such a reduction may represent progression of scar and "burnt out" disease.

Ahmadian et $\mathrm{al}^{8}$ studied the value of quantitative measures of FDG uptake using an SUV threshold determined by multiplying the left ventricular blood pool FDG $\mathrm{SUV}_{\max }$ by 1.5. Using this threshold, they calculated the volume of inflammation ("cardiac metabolic volume") as well as the volume-intensity product, which they termed "cardiac metabolic activity" (CMA). The study population included 31 patients (age $53.5 \pm 10.5$ years, $48 \%$ male, $87 \%$ biopsy proven extraCS) who underwent 38 cardiac PET scans. These studies were compared to negative control scans $(\mathrm{N}=10)$, acquired for oncologic indications. The authors showed that increased CMA was associated with lower ejection fraction as well as adverse clinical events. Their paper also provides useful insights regarding other aspects of cardiac PET.

The paper underscores the fact that $\mathrm{SUV}_{\max }$ is a poor measure of the total amount of FDG uptake as a scan with a small area of uptake may have a similar value as a scan with extensive uptake of the same intensity. In other words, the maximal intensity (SUVmax) does not reflect the extent of inflammation. However, $\mathrm{SUV}_{\max }$ was an independent predictors of a positive visual study, suggesting that this measure may be helpful in distinguishing pathologic "positive" studies from minimal uptake which is less likely to represent disease. Therefore, a visual interpretation, at times aided by assessment of $\mathrm{SUV}_{\max }$, may be sufficient for evaluating whether active inflammation is present or absent.

However, it is possible that quantification of the volume of inflamed myocardium (or CMA) may lead to a more accurate diagnosis, especially in cases in which the visual interpretation is less definitive. The paper had three patients with discordance of visual and quantitative assessment. Two patients (SUVmax 3.9 and $2.8 \mathrm{~g} /$ $\mathrm{mL}$; CMA 0.4 and $0.05 \mathrm{~g}$ ) were visually labeled as focal-on-diffuse uptake, however, quantitative assessment led to their reclassification as negative studies. These two cases illustrate the aforementioned limitation of "hot spot" imaging whereby mild defects may be amplified. The third discordant patient was visually interpreted as negative, however, quantitative assessment revealed mild focal uptake in the basal septum (SUV of $3.3 \mathrm{~g} / \mathrm{mL}$; CMA $8.1 \mathrm{~g}$ ). While a quantitative assessment led to reclassification in three patients, in the absence of a data on outcomes or diagnosis, it is difficult to conclude if these were accurate reclassifications. Another scenario where quantification may be useful is in $\sim 10 \%-15 \%$ of patients who have inappropriate suppression of FDG uptake from areas which are presumed to be normal. It remains to be seen whether quantitative techniques would be helpful in such instances.

The study by Ahmadian et al supports the fact that quantification of FDG uptake is important, however, the optimal technique for doing so is unknown. The advantage of the technique proposed by Ahmadian et al is that it accounts for variability in background noise, which may be important in the setting of serial exams, which have differences in background FDG activity. However, potential limitations of this approach are that even when meticulous care is taken to avoid the myocardium, due to cardiac and respiratory motion, the reproducibility of measuring left ventricular bloodpool SUV may be reduced. Future studies to quantify SUV uptake should compare absolute threshold techniques, such as those used by Osborne et $\mathrm{al},{ }^{4}$ with methods which utilize a variable threshold based on correcting for background activity.

The study by Ahmadian et al also reinforces the emerging role of cardiac PET for identifying patients with adverse cardiac events. ${ }^{3,9}$ Despite a low event rate, the authors were able to demonstrate that patients with adverse events had a higher measured CMA as well as a higher SUVmax. In contrast, our group was unable to detect any association between SUV max and adverse events. ${ }^{3}$ It is plausible that incorporating data on both the intensity and extent of inflammation, as well as also considering the extent and severity of resting myocardial 
perfusion defects, if present, would achieve the most robust risk assessment. However, since CS remains a rare condition, larger studies will be needed in order to demonstrate the potential incremental prognostic value of this approach. While Ahmadian et al included the endpoint of worsening heart failure, such events are less specific for CS. Future studies should focus on ventricular tachycardia and sudden cardiac death, particularly as the risk of such events may be most helpful in decision making regarding implantable cardiac defibrillator therapy.

In conclusion, the paper by Ahmadian et $\mathrm{al}^{8}$ offers an important method for quantifying disease activity and supports the increasing evidence that cardiac PET provides important diagnostic and prognostic information in patients with known or suspected CS. While further studies are needed to determine if quantification will improve the diagnostic and prognostic value over a visual read, inclusion of quantitative parameters of disease activity (which for the time being can use any method; e.g. Osborne et $\mathrm{al}^{4}$ or Ahmadian et $\mathrm{al}^{8}$ ) should be included in clinical reporting, as such data may improve the evaluation of disease activity thus guiding the role and duration of immunosuppressive therapy.

\section{References}

1. Iannuzzi MC, Fontana JR. Sarcoidosis: Clinical presentation, immunopathogenesis, and therapeutics. JAMA 2011;305:391-9.
2. Silverman KJ, Hutchins GM, Bulkley BH. Cardiac sarcoid: A clinicopathologic study of 84 unselected patients with systemic sarcoidosis. Circulation 1978;58:1204-11.

3. Blankstein R, Osborne M, Naya M, Waller A, Kim CK, Murthy VL, et al. Cardiac positron emission tomography enhances prognostic assessments of patients with suspected cardiac sarcoidosis. J Am Coll Cardiol 2014;63:329-36.

4. Osborne MT, Hulten EA, Singh A, Waller AH, Bittencourt MS, Stewart GC, et al. Reduction in ${ }^{18} \mathrm{~F}$-flurodeoxyglucose uptake on serial cardiac positron emission tomography is associated with improved left ventricular ejection fraction in patients with cardiac sarcoidosis. J Nucl Cardiol 2014;21:166-74.

5. Ohira H, Tsujino I, Ishimaru S, Oyama N, Takei T, Tsukamoto E, et al. Myocardial imaging with ${ }^{18} \mathrm{~F}$-fluoro-2-deoxyglucose positron emission tomography and magnetic resonance imaging in sarcoidosis. Eur J Nucl Med Mol Imaging 2008;35:933-41.

6. Tahara N, Tahara A, Nitta Y, Kodama N, Mizoguchi M, Kaida H, et al. Heterogeneous myocardial FDG uptake and the disease activity in cardiac sarcoidosis. JACC Cardiovasc Imaging 2010;3:1219-28.

7. Okumura $\mathrm{W}$, Iwasaki T, Toyama $\mathrm{T}$, Iso $\mathrm{T}$, Arai M, Oriuchi N, et al. Usefulness of fasting ${ }^{18} \mathrm{~F}$-FDG PET in identification of cardiac sarcoidosis. J Nucl Med 2004;45:1989-98.

8. Ahmadian A, Brogan A, Berman J, Sverdlov AL, Mercier G, Mazzini M, et al. Quantitative interpretation of FDG PET/CT with myocardial perfusion imaging increases diagnostic information in the evaluation of cardiac sarcoidosis. J Nucl Cardiol 2014. doi: 10.1007/s12350-014-9901-9

9. Mc Ardle BA, Birnie DH, Klein R, de Kemp RA, Leung E, Renaud $\mathrm{J}$, et al. Is there an association between clinical presentation and the location and extent of myocardial involvement of cardiac sarcoidosis as assessed by ${ }^{18} \mathrm{~F}$-flurodoexyglucose positron emission tomography? Circ Cardiovasc Imaging 2013;6:617-26. 